\title{
Developing affective mental imagery stimuli with multidimensional scaling
}

\author{
Matthew J. Facciani ${ }^{\text {国, a }}$ \\ a Department of Psychology, University of South Carolina
}

\begin{abstract}
The goal of this paper is to provide an example of how multidimensional scaling (MDS) can be used for stimuli development. The study described in this paper illustrates this process by developing affective mental imagery stimuli using the circumplex model of affect as a guide. The circumplex model of affect argues that all emotions can be described in terms of two underlying primary dimensions: valence and arousal (Russel, 1980). We used MDS to determine if affective mental imagery stimuli obtained from verbal prompts could be separated by arousal and valence to create four distinct categories (high -positive, low-positive, high-negative, and low-negative) as seen in other stimuli. 60 students from the University of South Carolina participated in the first experiment to evaluate three sets of stimuli. After being analyzed using MDS, selected stimuli were then assessed again in a second experiment to validate their robust valence and arousal distinctions. The second experiment was conducted with 34 subjects to validate 40 of the best stimuli from experiment 1 . It was found that mental imagery stimuli can produce a reliable affective response for the dimensions of valence and arousal and that MDS can be an effective tool for stimuli development.
\end{abstract}

Keywords " multidimensional scaling, stimuli development, mental imagery, affect, SYSTAT

$\equiv$ facciani@email.sc.edu

\section{Introduction}

Knowing which statistical methods to use can greatly assist the vital (yet tedious) process of stimuli development for psychological research. This study aimed to illustrate how multidimensional scaling (MDS) could assist with the development of affective mental imagery stimuli. MDS revealed if the present stimuli were congruent with the circumplex theory of affect (Russel, 1980). This process can be applied to a wide range of potential stimuli.

\section{Core Affect}

The term affect has been used in the behavioral sciences since the early days of Wilhelm Wundt. Wundt characterized affect as a feeling state vital to cognition. This feeling state could be altered from a variety of experiences. His example was that "the unpleasureableness of a toothache, of an intellectual failure, and of a tragic experience are all regarded as identical affective contents" (1897, p.85). Thus, how people felt about things could be altered in the same way regardless of the stimulus they were presented with. Wundt's conceptualization of affect was quite congruent with contemporary psychologists. Affect is presently regarded as a mental state in response to a stimulus which can be pleasant or unpleasant with some degree of arousal (Barrett \& Bliss Moreau, 2009;
Russell 2003). Valence can be described as how positive or negative an emotion is and arousal can be described by how strong an emotion is (Russell, 2003).

Core affect then characterizes emotion as an internal and consciously accessible state which is comprised of an integral blend of valence and arousal (Brosch, Pourtoise, \& Sander, 2010; Russel, 2003). Core affect can be altered by different stimuli. The greater the change in core affect from the stimulus, the stronger affective quality the stimulus has (Brosch, Pourtoise, \& Sander, 2010; Russel, 2003). A person's core affect can be understood from a linear combination of the dimensions of valence and arousal (Barrett \& Russel, 1999; Feldman, 1995; Russel, 1980; Russel \& Barrett, 1999). The affective state may have positive valence and high arousal differing it from an affective state that elicits positive valance and low arousal. For example, the affective states of calm and excited would be considered positive emotions; however, they would differ significantly on arousal.

A common way to scientifically study core affect is to present a participant with an affective stimulus, and document the ensuing affective response. Picture, word, sound, and smell stimuli have been shown to reliably elicit affective responses derived from the judgments participants make on them (Alaoui-Ismailli, et al., 1997; 


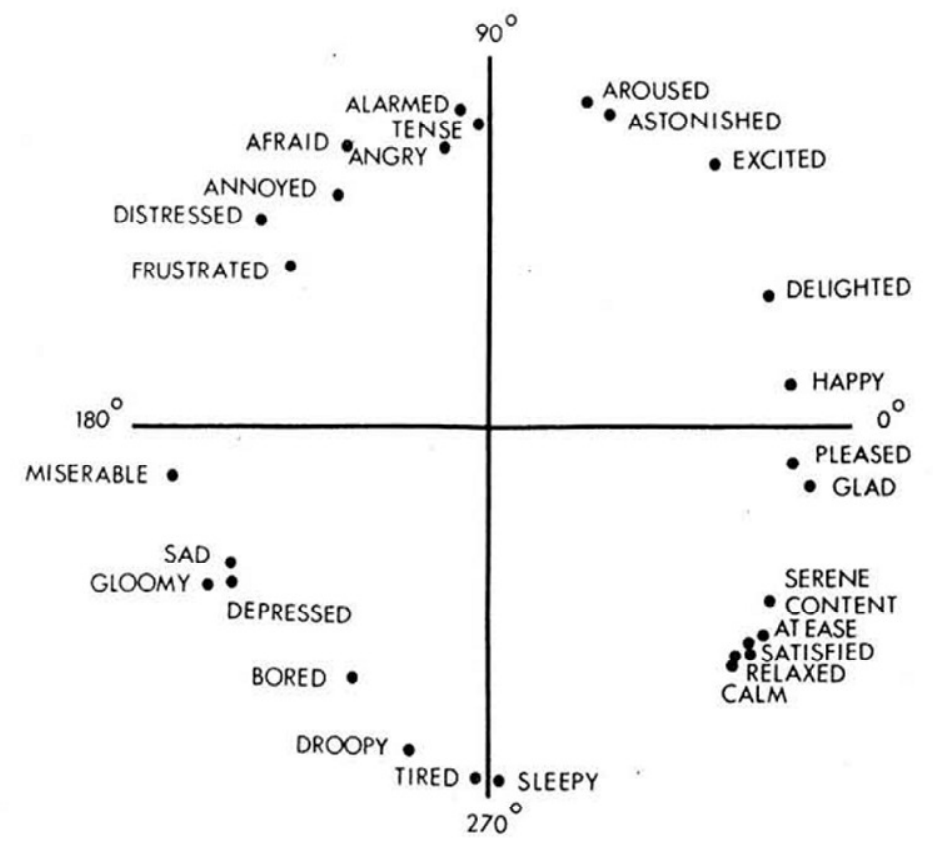

Figure 1 - A Circumplex Model of Affect from 28 emotional words displaying valence and arousal (Russel, 1980).

Jackson et al., 2000; Roberts \&Wedell, 1994; Zentner, Grandjean, \&Scherer, 2008;). The variability in judgments of affective stimuli have been shown to result in a circumplex-like pattern which fostered the concept of the circumplex model of affect.

\section{The Circumplex Model of Affect}

Mapping affective state ratings on a lower dimensional space is a way to convey and visualize valence and arousal. Calm and excited would have similar dimensions of valence, but they would differ significantly on arousal, which would make them far apart on the arousal dimension. Varying affective states can be represented by their location on the two dimensional space, which ultimately lead to a circumplex-like figure (e.g. located on the circumference of a circle; see Figure 1). Hence, the name of this approach of studying affective states; the circumplex model of affect (Barrett \&Russel, 1999; Feldman, 1995; Russel\& Barrett, 1999; Russel, 1980; Russel 2003).

The circumplex model of affect has been a leading contemporary model for studying affective states because it provides a more flexible alternative to studying emotions in a categorical fashion (i.e. anger, disgust, happy). By taking into account both valence and arousal the circumplex model considers the underlying structure of how an emotion can be represented on a lower dimensional space (Posner et al., 2005; Russell, 1980). Many different types of stimuli have been studied using this model such as pictures, sounds, and words. (Jackson et al., 2000; Roberts \&Wedell, 1994; Zentner, Grandjean, \& Scherer, 2008). However, all of the stimuli types require some form of perception. It is presently unknown whether this circumplex pattern can be found in a stimuli type not requiring perception, such as mental imagery.

\section{Mental Imagery}

Mental imagery has been researched extensively in psychology research (Kosslyn 1988; Pylyshyn 1973) and recently has been studied as an emotional stimulus (Holmes et al., 2008; Holmes \& Mathews, 2005). Mental imagery can be characterized as a mental representation without a perceptual cue (Kosslyn, 1988). Previous research suggests that there are many similarities and relationships between the imagery of a picture and the picture itself (Ganis, 2004; Kosslyn, 1988).

It was proposed by Holmes and Mathews (2005) that mental imagery and emotion may have a special relationship in the brain because of how mental imagery stimuli can produce more anxiety compared to word stimuli. In a subsequent study, Holmes and colleagues (2008) investigated how mental imagery may actually elicit stronger overall emotional 
responses in comparison to verbal processing in several emotions. These researchers concluded this may be due to (i) the sensitivity of emotional brain regions to imagery, (ii) how similar imagery is to perception, (iii) and the influence of emotional autobiographical memories. While emotional mental imagery has been studied extensively, it is unknown if mental imagery can produce affective states similarly seen in word, sound, and picture stimuli. Multidimensional scaling could be a useful statistical method to determine if mental imagery could produce an affective circumplex pattern.

\section{Multidimensional scaling}

MDS is an exploratory data analysis technique that allows the researcher to visualize the structure of the data (Torgerson, 1952). MDS conceptualizes similarities between objects as distances in lower dimensional space (Kruskal \& Wish, 1978). The mathematical objective of MDS is to find a space of low dimensionality in which objects can be placed so that the Euclidian distance between them match perceived similarity expressed by the participants (Shepard, 1962). The map comprised of all the points is specified by a corresponding representative function. This function states how the distances should be ideally related. The solution is found by the ideal configuration which satisfies the function as best as possible (Groenen \& van de Velden, 2004). This solution is then evaluated by a measure that assesses how good or bad the fit is. This measure is called stress which is a normed sum-of-squares of errors. The more error there is between the observed data and the proposed map, the higher the stress. Generally, a stress value lower than .05 is considered excellent (Kruskal \& Wish, 1978). To show the goodness of fit for MDS, the Rsquared value can explain how much of the variance in the data is accounted for by the corresponding distances. R-squared and stress are only two of the various techniques available to analyze circumplex models (Tracey, 2000).

MDS is a great way to visualize the data from a single matrix. This single matrix is a matrix of similarity (or dissimilarity) such that the entry in row $i$, column $j$ indicates to what level the subject felt that item $i$ was similar (or dissimilar) to item $j$. If the researcher wants to compare multiple matrices of data and explore individual differences, different techniques must be used. To examine a lower dimensional representation of the individual matrices, Individual Differences Multi-
Dimensional Scaling (INDSCAL) (Carroll \& Chang, 1970) can be used to find individual differences which is vital for stimuli development. This technique creates a prototype of the averaged matrices first. After that, each individual matrix is compared to the prototype to see how different it is from the common configuration. If a matrix has to stretch itself greatly to fit the prototype, then it does not have a good fit and is considered to be dissimilar. Thus, for INDSCAL, a low stress value for a particular matrix would suggest it is quite similar to the common configuration. Generally, increasing the number of matrices will increase the stability of the prototype of averaged matrices (Carroll \& Chang, 1970). Giguère (2006) offers a great guide for collecting and analyzing data in multidimensional scaling experiments using SPSS. Jaworska \& Chupetlovska-Anastasova (2009) discuss how multidimensional scaling can be used for various psychological domains, but do not discuss how it can be used for stimuli development. The present study aims to illustrate how MDS and INDSCAL can be used for affective mental imagery stimuli development.

\section{Methods}

\section{Participants}

Ninety four students from the University of South Carolina were recruited via Sona Systems (participant recruitment software) and received partial course credit to participate in the experiments. Sixty of these participants participated in the first experiment and 34 participated in the second experiment. Participants were given an informed consent form, in accordance with the institutional review board at the University of South Carolina, and completed the experiment in a controlled room on a computer.

\section{Materials and Procedure}

The initial set of mental imagery stimuli was generated from the four quadrants of the valence-by-arousal affective space: positive high, positive low, negative high and negative low. One hundred and twenty total stimuli were created by the author and other lab members. Participants were asked to imagine various objects and scenarios, for example, 'imagine a winning lottery ticket.' For each trial, a participant had 4 seconds to imagine the object or scenario, then make a response on a computer indicating the degree to which they felt positive, happy, relaxed, calm, excited, anxious, angry, negative, sad, and disgusted, with 1 


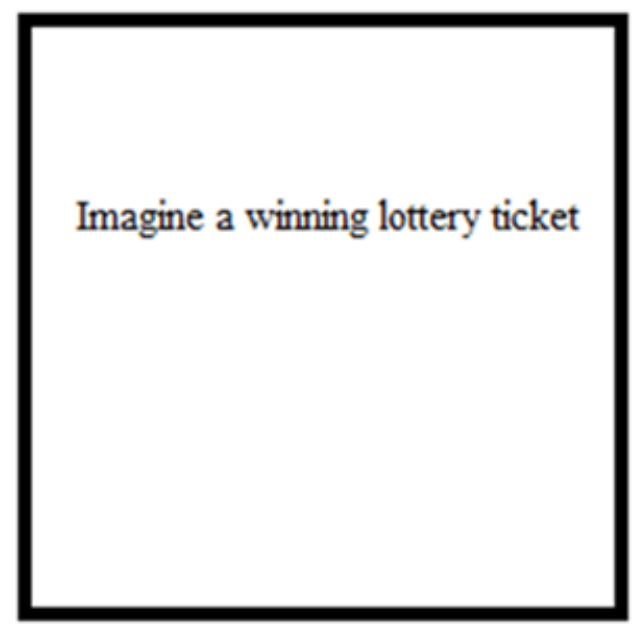

4s

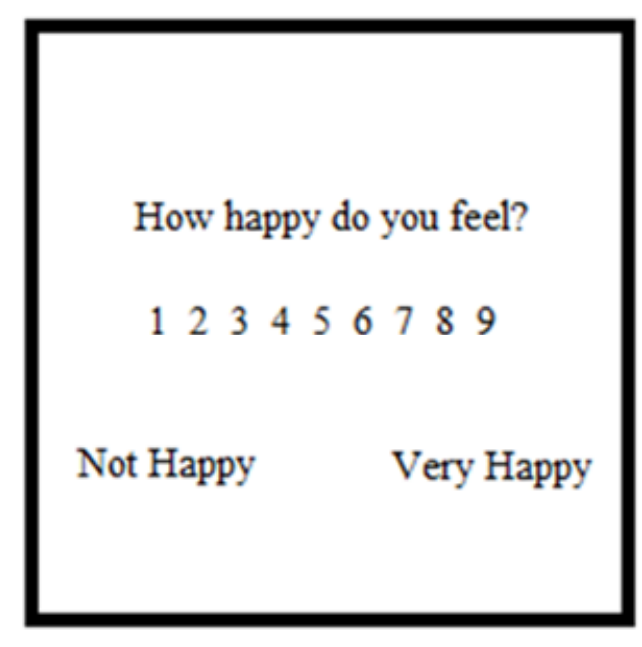

Response Time

Figure 2 - Illustration of experimental task. Stimuli were presented on a computer screen with E-PRIME for $4 \mathrm{~s}$ followed by the participant's response to a rating scale.

corresponding to "not at all" and 9 corresponding to "very much" (Figure 2). Imagery stimuli were presented 10 separate times each, to obtain a rating on each of 10 scales. Stimuli were presented using E-Prime 2.0 software (Psychology Software Tools, Pittsburgh, PA).

\section{Experiment 1}

Ratings for the 120 phrases were collected to examine the variability in the ratings among stimuli. of the 60 participants who participated in this study, 20 participants were run for each stimuli set consisting of 40 phrases. Segmenting the 120 phrases into three sets was done to shorten the experiment and reduce test fatigue (each participant only rated 40 stimuli). The stimuli with the most extreme valance and arousal ratings revealed by the MDS results were compiled into a new stimuli set for experiment 2.

\section{Experiment 2}

The goal of collecting data from this set was to validate the affective responses to the selected stimuli and create a validated subset of stimuli with strong differences on valence and arousal ratings. Participants in experiment 2 completed the same procedure with a subset of stimuli from experiment 1 . By doing this, a subset of categorical affective stimuli from experiment 1 was created. This selected subset of the 40 most extreme stimuli from experiment 1 formed four distinct categories (high positive, low positive, high negative, low negative). The selection was made by judging which stimuli were most extreme in each quadrant (i.e. high positive stimuli would be in the top right of the MDS solution).

\section{Data Preprocessing}

Each participant's data from E-Prime was transferred in Excel to create a spreadsheet for all of the data per each experiment. The rows represent the participant's rating scores for each scale and the columns represent the stimuli (i.e. "kitten" is LP5). Each stimuli had a label assigned (HN for high negative, HP for high positive, LN for low negative, and LP for low positive) as well as an ID number. Finally, the excel data is taken from the Excel file (see Figure 3) and transferred into SYSTAT.

Listing 1 - SYSTAT code for importing the Excel file into SYSTAT.

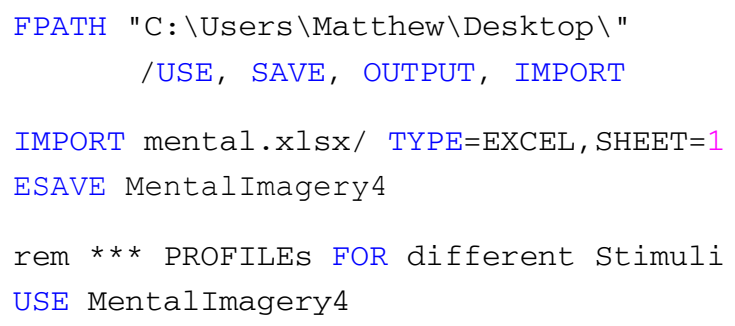

Three participants were removed from final analysis of experiment 2. We removed these participants because 3 out of the 4 participants with the highest stress value were responding with one answer in the 


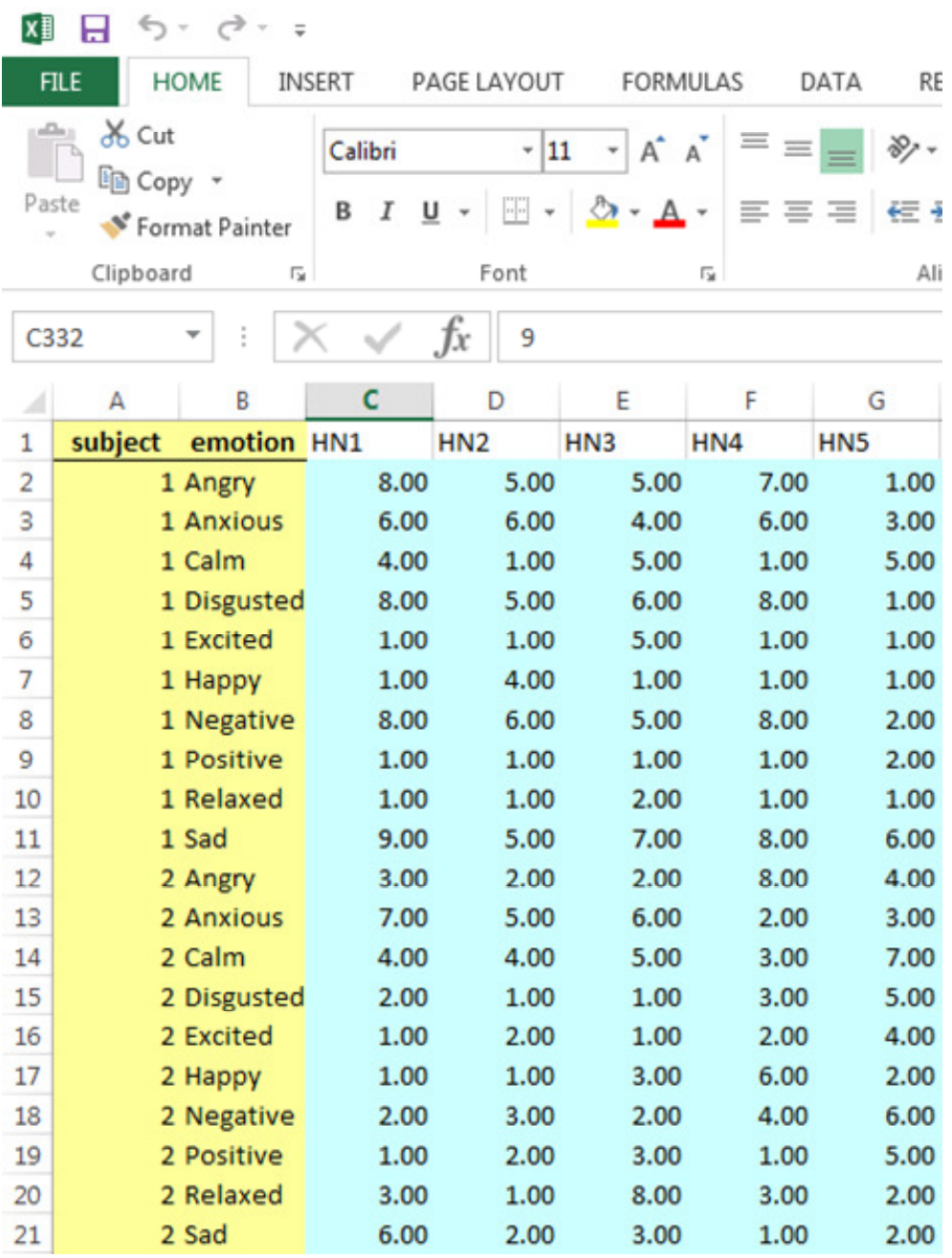

Figure 3 - Excel file with E-prime data showing first two participants (subject column), rating scales (emotion column), and the first five high negative stimuli shown in the screen capture (coded HN1-HN5).

ratings for the majority of the experiment and were probably not giving a strong effort. The other participant with a high stress value did have a variety of responses so we felt their data was still legitimate.

\section{Correlation Matrices}

Pairwise correlations were computed from the participant's judgment ratings and the affective imagery stimuli. These pairwise correlations were then used to create a correlation matrix of ratings for each participant (see Figure 4). These correlation matrices determine the relationship between the affective ratings and measures the similarity between the stimuli. The data were analyzed as similarities, which produced data matrices. The number of data matrices corresponds to the number of subjects. For example, there were 34 data matrices produced during MDS in
Experiment 2 because Experiment 2 had 34 subjects. The correlation matrices of subject data were then appended to prepare for INDSCAL. To analyze similarity of stimulus ratings, Individual Differences Multi-Dimensional Scaling (INDSCAL) (Carroll \& Chang, 1970) was conducted. See Listing 2 for example code.

\section{Multidimensional scaling methods}

MDS visualizes dissimilarities between objects as distances in lower dimensional space. The objects would be the mental imagery stimuli for the present study. Similarity among imagery stimuli is then computed from the affective judgment ratings. The relationship between the affective ratings was created by a correlation matrix and is the measure of similarity between the objects. Each object can be represented on a two dimensional space by a single point. The present 


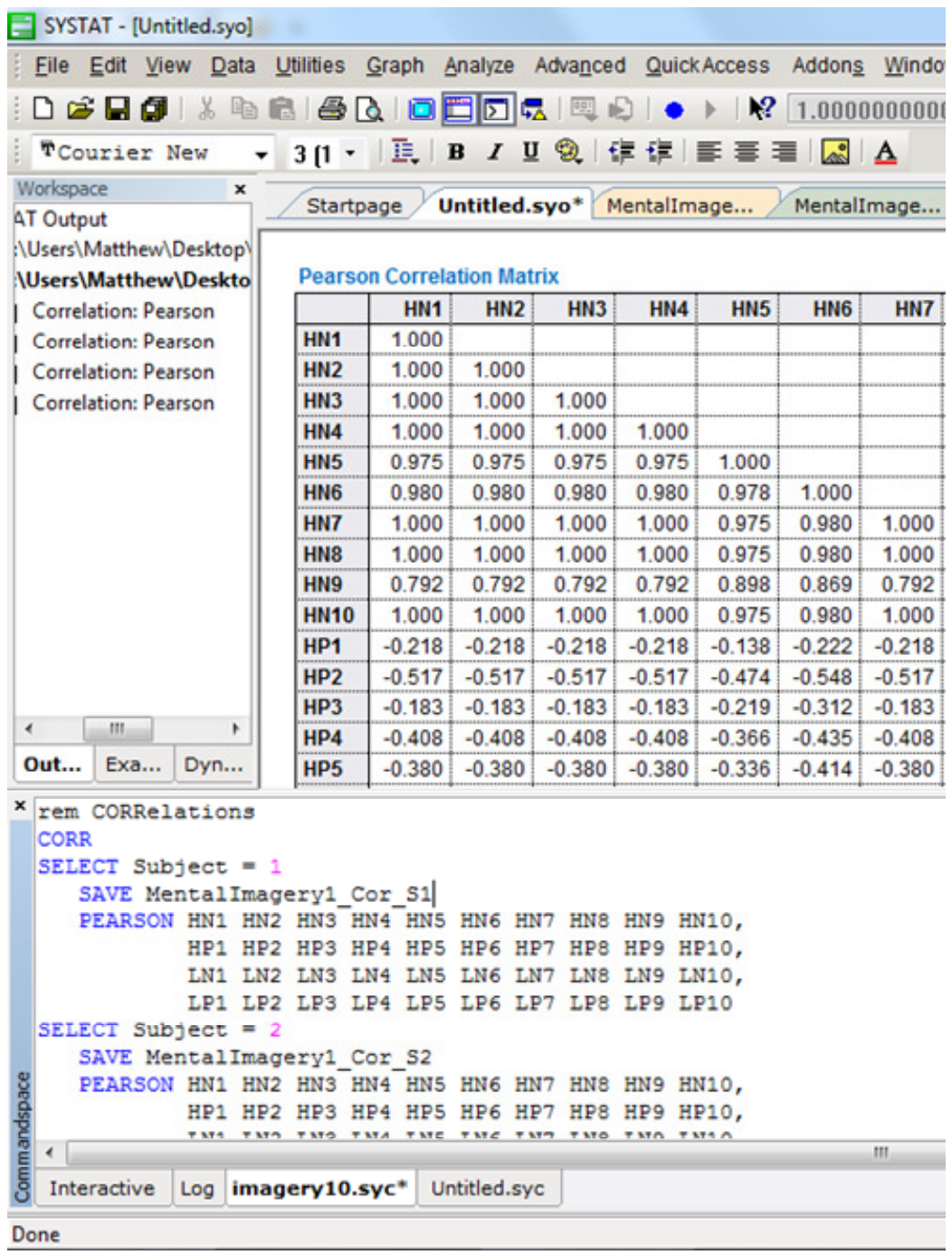

Figure 4 = Pearson correlation matrix produced in SYSTAT from participant's judgment ratings and affective imagery stimuli.

study would aim for a configuration which mirrors the circumplex model of affect and the farther two points are on the space, the more dissimilar they are. Importantly, each point in the lower dimensional space represents each stimulus making it quite easy to visually determine how similar and different the stimuli are from each other. The final step is to use the appended matrices for monotonic MDS and ultimately INDSCAL using the Kruskal method. See Listing 3.

Evaluation of stimuli can be done visually or looking at the highest (or lowest) distances from the coordinates in two dimensions. The appendix provides the example output of the MDS and INSCAL solution.

The MDS methods for both Experiment 1 and 2 were identical. Affective judgment ratings for each stimuli were compiled in an Excel spreadsheet from the E- prime experiment. The Excel spreadsheet was then loaded into SYSTAT (Systat Software, Inc., 2007), which had each subject's ratings of all the stimuli. SYSTAT software was used to conduct the INDSCAL to determine the individual differences in the ratings of stimuli to find which stimuli were consistently rated in a particular area of the circumplex. For INDSCAL, a low stress value for a particular matrix would suggest it is quite similar to the common configuration. The strength of association between the participant's original data and the overall values predicted by the INDSCAL model can be represented by R-squared. So if a single participant's data had a high stress value and low R-squared, the researchers could conclude that their affective ratings were not consistent with the aggregate ratings from the rest of the participants. We 
Listing 2 - SYSTAT code for running correlations on the affective mental imagery stimuli data. The correlation matrices were then appended to prepare for INDSCAL. Code for only four participants is shown as an example for clarity purposes. Additional participant data can be added by following the same procedure.

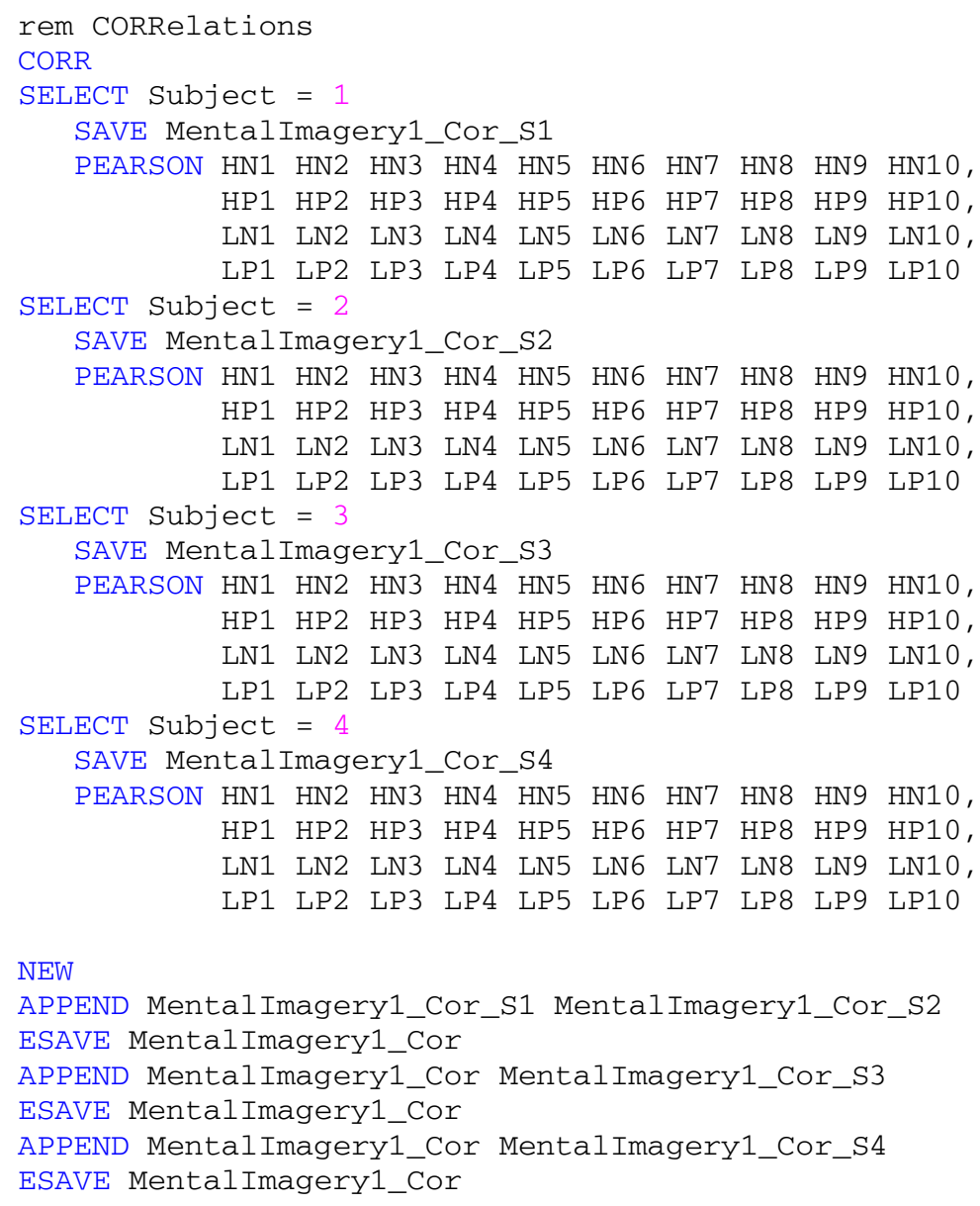

Listing 3 - SYSTAT code for running MDS on the affective mental imagery stimuli data.

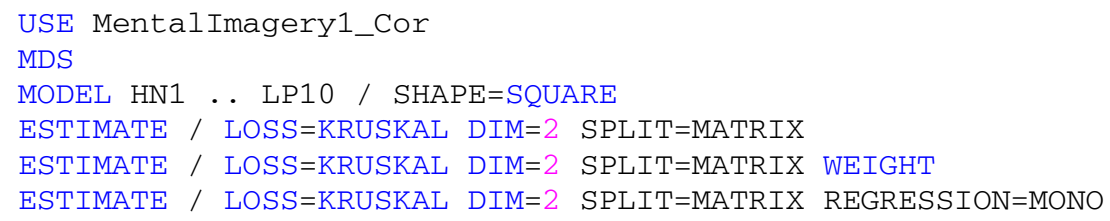

felt that R-squared and stress values were sufficient for our study, but as noted above there are many other techniques to analyze circumplex models (Tracey, 2000).

\section{Results}

A circumplex structure emerged from the mental imagery ratings from experiment 1 (see Figure 5). Valence was separated very well overall with the exception of 'rainy day' being neutral in valence in experiment 1 . Arousal was not separated as well with many stimuli falling in the middle of the space (see Figure 5). Based on the configuration resulting from Experiment 1, 40 stimuli with valence and arousal ratings on the extremes of the valence-arousal space (e.g. in the outside corner of the four quadrants) were selected for experiment 2. Evaluation of stimuli from the first experiment was done visually and by choosing the highest (or lowest) distances from the coordinates in two dimensions.

Experiment 2 showed very good separation of valence and arousal and validated most of the stimuli, 

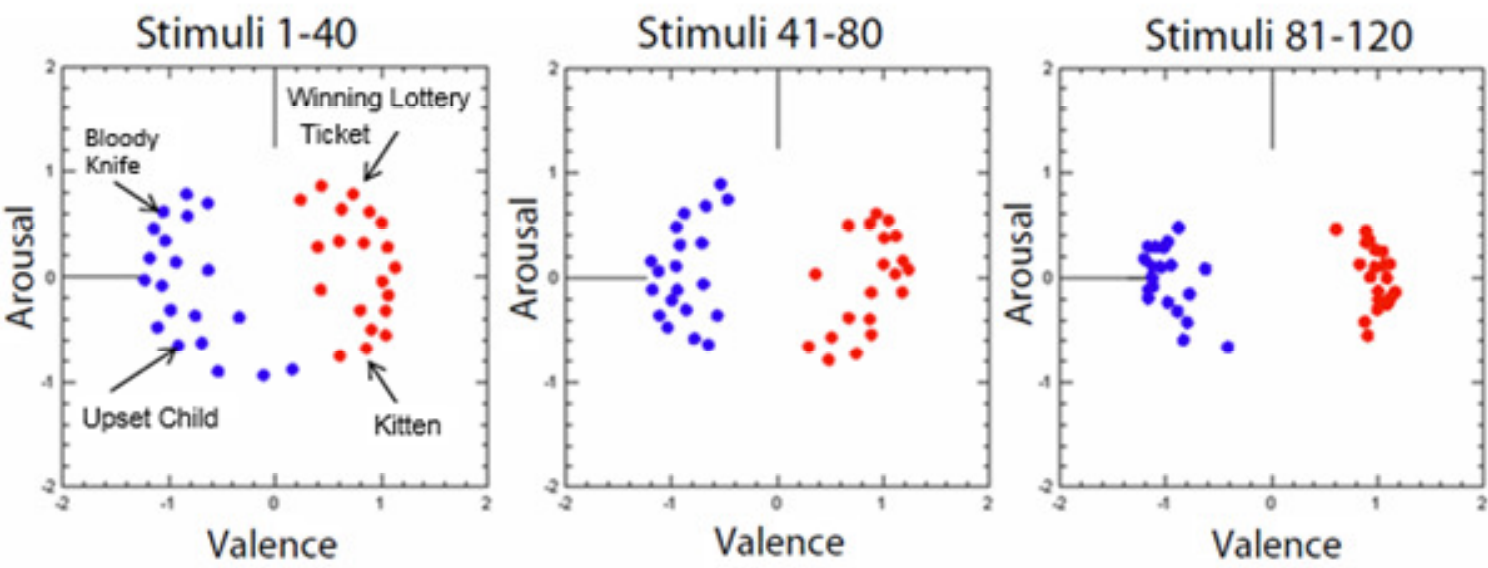

Positive

Negative

Figure 5 - Experiment 1: A two-dimensional multidimensional scaling solution for three stimuli sets $(\mathrm{N}=20)$. Each dot represents a short phrase whereas blue represents negative stimuli and red represents positive. The collection of dots produces a clear representation of the circumplex on the dimensions of arousal and valence. The stimulus "rainy day" can be seen close to the center coordinate in the second panel, which suggests neutral valence and arousal.

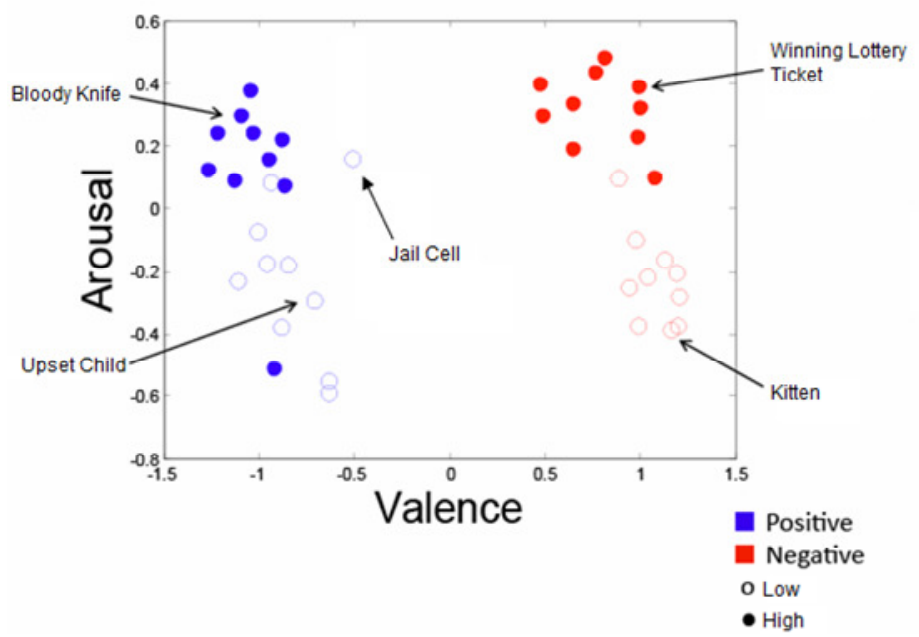

Figure 6 - Experiment 2: A multidimensional scaling solution for the validated stimuli set $(\mathrm{N}=34)$. Each dot represents a short phrase. The collection of dots produces a clear representation of the circumplex on the dimensions of arousal and valence. Blue represents negative stimuli, whereas red represents positive. The scales on this figure represent arbitrary units of distance. The "a bowl of rotten eggs" stimulus can be seen as the filled in blue dot in the lower left quadrant.

but there were a few exceptions (see Figure 6). 'A bowl of rotten eggs', for example, was more similar to high arousal stimuli in the first experiment, but was more similar to low arousal stimuli in the second experiment. Additionally, 'jail cell' were low negative in the first experiment, but became high negative in the second experiment. 'Unopened present' also changed arousal from the first experiment as it moved from low positive to slightly high positive. Profile plots revealed which affective rating scales contributed to the location of a point on the space. For example, 'bloody knife' had high ratings for the 'negative' and 'anxious' scales across participants which contrasted to 'kitten' having low ratings in the 'negative' and 'anxious' scales. Conversely, 'bloody knife' had low ratings on the 'calm' and 'positive' scales, whereas 'kitten' had high ratings 


\section{Imagine a bloody knife}

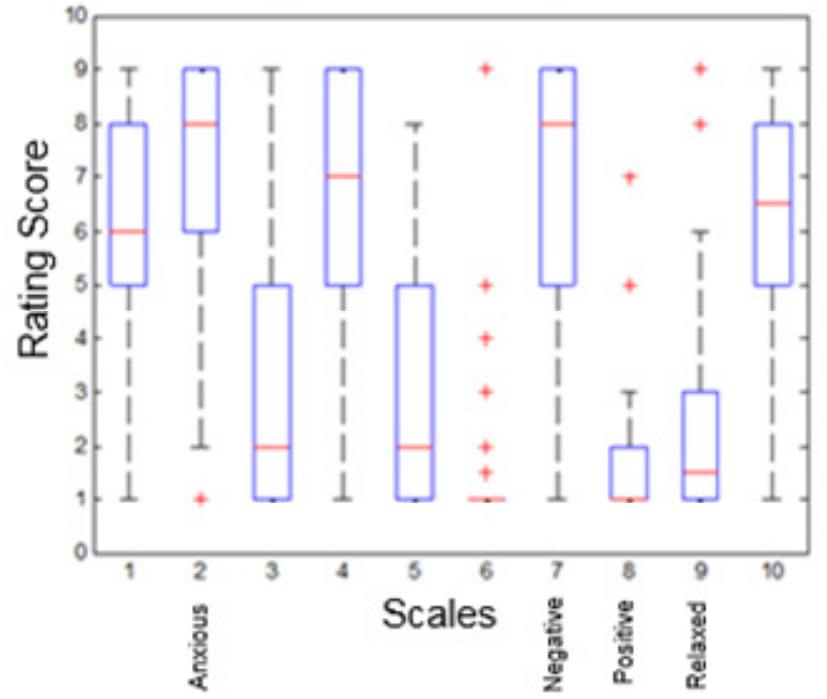

Imagine a kitten

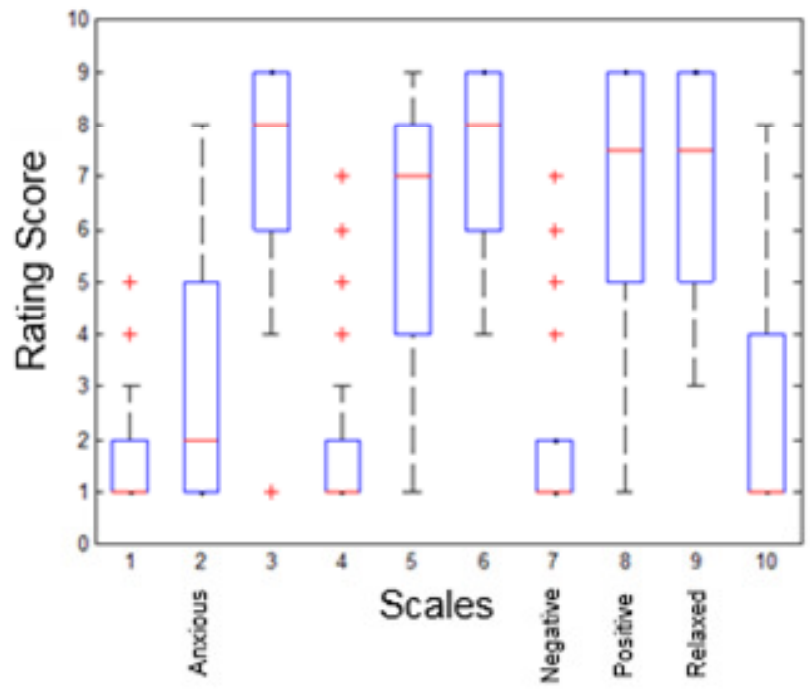

Figure 7 - Profile plots for stimuli ratings in experiment 2. The $Y$ axis represents the different scales used to determine affect and arousal. The $\mathrm{X}$ axis represents the participant's rating score of the stimuli from 1-10.

on those same scales (see Figure 7).

A two dimensional solution of the multi-dimensional scaling had low stress and was straightforward to plot as most of the variance could be explained from two dimensions. The average R-squared was 0.606 with outliers (the three participants who mostly responded with one answer) and 0.653 without them. The average stress value was 0.289 and 0.277 without outliers.

\section{Discussion}

The goals of the current study were to develop short phrase mental imagery stimuli and determine if these stimuli could elicit similar affective responses to those elicited from stimuli required perception. Using MDS, it was found that mental imagery can produce a reliable affective response, and most of the variability in judgments is accounted for by valence and arousal. Short phrase mental imagery could elicit stronger emotional responses than other stimuli (Holmes et al., 2008; Holmes \& Mathew, 2005) and this study suggests that they produce reliable affective states as well. Because this study demonstrates that mental imagery stimuli can elicit a reliable affective response, a future study could collect more data to create a normed set of affective imagery stimuli as seen with words (Bradley \& Lang, 1999), pictures (Lang, Bradley, \& Cuthbert, 1997), and sounds (Bradley \& Lang, 1999) stimuli.

Future research could assess how personality and mood variables affect the judgements of affective imagery stimuli. For example, anxious participants may rate negative stimuli more strongly. One limitation of this study is that no personality or mood variables were recorded. Additionally, this study had participants imagine the scenarios for four seconds, but a longer or shorter time period may alter the structure of data. Also, this study had several stimuli that changed locations in the affective space between the two experiments. Additional iterations of the experiment may make those stimuli more stable, but we would suggest removing items that constantly change in their arousal and valence.

Future research can also investigate how affective states are created in different types of sensory modalities (i.e. visual cues vs auditory cues). This would be interesting to look at because researchers tend to predominantly use visual mental imagery, but Zvyagintsev and colleagues (2013) found that there are different brain networks for visual and auditory imagery. Investigating how affect is involved in these networks would be an exciting addition to understanding the neural processes of imagery across modalities.

Importantly, this study illustrated the process of using MDS and INDSCAL for the development of stimuli. The same process can be used for any stimuli set. The researchers can take the best stimuli (i.e. the stimuli which are most separated in the relevant dimensions) from a series of experiments to develop a single strong 
set of stimuli. The circumplex model of affect provides an easy map to measure stimuli, but future studies can use MDS to visualize the similarities of stimuli from any theory. The key part of the present study is that it describes how using multidimensional scaling in a series of experiments can develop stimuli. We hope that future researchers can employ this technique in their own stimuli development.

\section{Authors' notes and acknowledgments}

I would like to thank Jongwan Kim, Mary-Catherine Newell, Neha Jaggi, and Arjamand Sami for their help creating stimuli and collecting data for this study. Correspondence to: Matthew Facciani, Department of Psychology, University of South Carolina, Columbia, South Carolina, 29208, USA.

\section{References}

Alaoui-Ismaïli, O., Robin, O., Rada, H., Dittmar, A. and Vernet-Maury, E. (1997) Basic emotions evoked by odorants: comparison between autonomic responses and self-evaluation. Physiological Behavior, 62, $713-720$.

Barrett, L.F., Bliss-Moreau, E., 2009. Affect as a psychological primitive. Advances in Experimental Social Psychology, 41, 167-218.

Barrett, L. F., \& Russell, J. A. (1999). Structure of current affect. Current Directions in Psychological Science, 8, 10-14.

Bradley, M. M., \& Lang, P. J. (1994). Measuring emotion: The self-assessment manikin and the semantic differential. Journal of Behavioral Therapy and Experimental Psychiatry, 25, 49-59.

Bradley, M. M., \& Lang, P. J. (1999). International affective digitized sounds (IADS): Stimuli, instruction manual and affective ratings (Tech. Rep. No. B-2). Gainesville, FL: The Center for Research in Psychophysiology, University of Florida

Bradley, M. M., \& Lang, P. J. (1999). Affective norms for English words (ANEW): Instruction manual and affective ratings (Tech. Rep. No. C-1). Gainesville, FL: University of Florida, The Center for Research in Psychophysiology.

Brosch T., Pourtois G., \& Sander D. (2010). The perception and categorisation of emotional stimuli: A review. Cognition \& Emotion, 24(3): 377-400.

Carroll, J. D., \& Chang, J. J. (1970). Analysis of individual differences in multidimensional scaling via an $\mathrm{N}$ way generalization of "Eckart-Young" decomposition. Psychometrika, 35(3), 283-319.
Feldman, L. A. (1995). Valence focus and arousal focus: Individual differences in the structure of affective experience. Journal of Personality and Social Psychology, 69, 153-166.

Ganis, G., Thompson, W. L., and Kosslyn, S. M. (2004). Brain areas underlying visual mental imagery and visual perception: an fMRI study. Cognitive Brain Research, 20, 226-241.

Giguère, G. (2006). Collecting and analyzing data in multidimensional scaling experiments: A guide for psychologists using SPSS. Tutorials in Quantitative Methods for Psychology, 2(1), 27-38.

Groenen, P., \& VandeVelden, M. "Multidimensional Scaling,"(2004). Econometric Institute Report.

Holmes, E.A. \& Mathews, A. (2005). Emotion, 5(4), 489497.

Holmes, E.A., Mathews, A., Mackintosh, B., \& Dalgleish, T. (2008). Emotion, 8(3), 395-409.

Jackson, D. C., Malmstadt, J. R., Larson, C. L. and Davidson, R. J. (2000), Suppression and enhancement of emotional responses to unpleasant pictures. Psychophysiology, 37, 515-522.

Jaworska, N., \& Chupetlovska-Anastasova, A. (2009). A review of multidimensional scaling (MDS) and its utility in various psychological domains. Tutorials in Quantitative Methods for Psychology, 5(1), 1-10.

Kosslyn, S. M. (1988). Aspects of a cognitive neuroscience of mental imagery. Science, 240, 16211626. Reprinted in B. J. Baars, W. P. Banks, and J. B. Newman (Eds.), Essential sources in the scientific study of consciousness. Cambridge, MA: MIT Press. pp. 457-468.

Kruskal, J. B., \& Wish, M. (1978). Multidimensional scaling (Vol. 11). Sage.

Lang P. J., Bradley, M., M., \& Cuthbert, B. N. (1997). International Affective Picture System (IAPS): Technical Manual and Affective Ratings (National Institute of Mental Health Center for the Study of Emotion and Attention, Gainesville, FL).

Lang, P. J., Bradley, M. M., \& Cuthbert, B. N. (1997). Motivated attention: Affect, activation, and action. In P. J.Lang, R. F. Simons, \& M. T. Balaban (Eds.), Attention and orienting: Sensory and motivational processes (pp.97-135). Hillsdale, NJ: Erlbaum.

Pylyshyn, Z. W. (1973). What the mind's eye tells the mind's brain: a critique of mental imagery. Psychological Bulletin, 80, 1-24

Posner, J., Russell, J., Peterson, B.S., 2005. The circumplex model of affect: an integrative approach to affective neuroscience, cognitive development, 
and psychopathology. Developmental Psychopathology, 17, 715-734.

Roberts, J.S., \& Wedell, D.H. (1994). Context effects on similarity judgments of multidimensional stimuli: Inferring the structure of the emotion space. Journal of Experimental Social Psychology, 30, 1-38.

Russell, J., 1980. A circumplex model of affect. Journal of Personality and Social Psychology, 39, 1161-1178.

Russell, J. A., \& Barrett, L. F. (1999). Core affect, prototypical emotional episodes, and other things called emotion: Dissecting the elephant. Journal of Personality and Social Psychology, 76, 805-819.

Russell, J. A. (2003). Core affect and the psychological construction of emotion. Psychological Review, 110, 145-172.

Shepard, R. N. (1962). The analysis of proximities: Multidimensional scaling with an unknown distance function. I. Psychometrika, 27(2), 125-140.

Systat Software, Inc. (2007). SYSTAT 12 statistics.
Torgerson, W. S. (1952). Multidimensional scaling: I. theory and method. Psychometrika, 17, 401-419

Tracey, T. J. G. (2000). Analysis of circumplex models. In H. E. A. Tinsley, \& S. D. Brown (Eds.), Handbook of applied multivariate statistics and mathematical modeling (pp. 641-664). New York: Academic Press.

Wundt, W. (1998b). Outlines of psychology (C.H. Judd, Trans.). Bristol, UK: Thoemmes Press (Original work published 1897).

Zentner, M., Grandjean, D., \& Scherer, K. R. (2008). Emotions evoked by the sound of music: characterization, classification, and measurement. Emotion, 8(4), 494.

Zvyagintsev, M., Clemens, B., Chechko, N., Mathiak, K. A., Sack, A. T., \& Mathiak, K. (2013). Brain networks underlying mental imagery of auditory and visual information. European Journal of Neuroscience, 37(9), 1421-1434.

\section{Appendix: SYSTAT output of coordinates of stimuli and plot of MDS solution}

Each dot represents where the stimuli falls on the multidimensional space.

\section{Configuration}

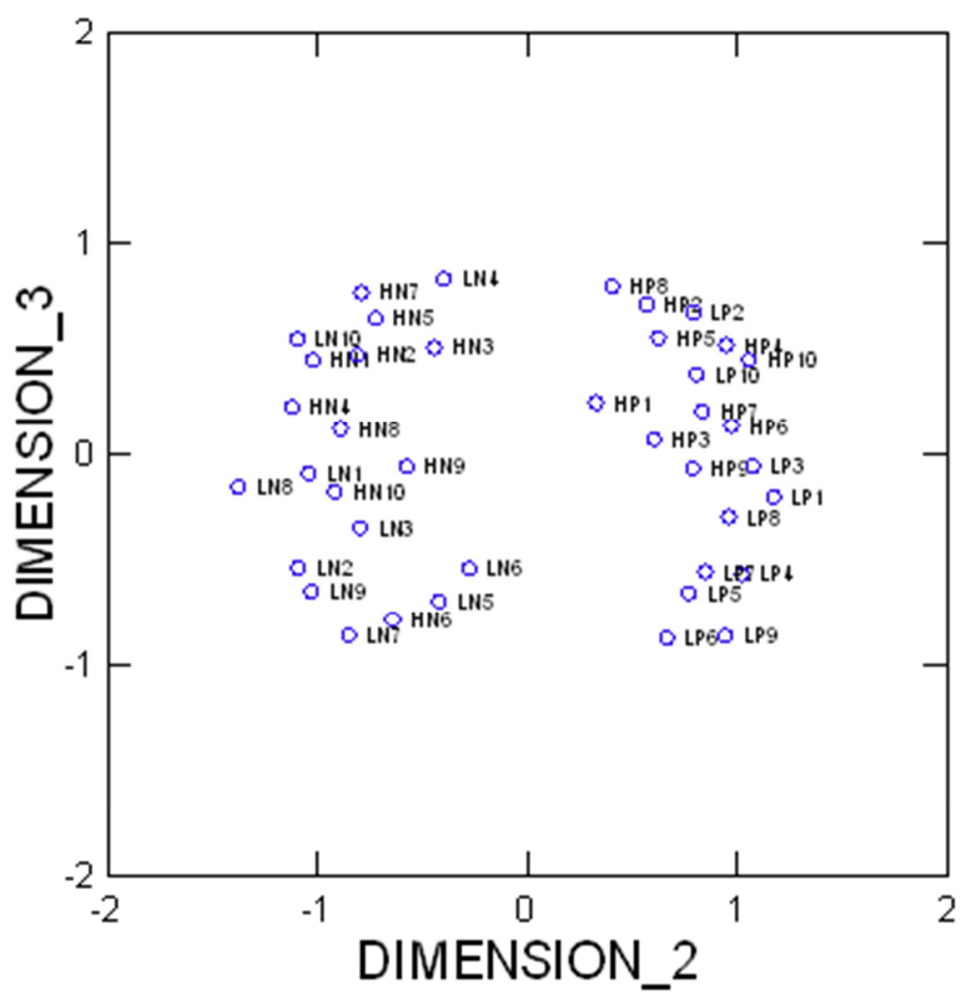

The Quantitative Methods for Psychology 


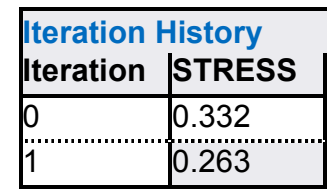

Stress of Final Configuration : 0.263

Proportion of Variance (RSQ) : 0.606

\section{Shepard Diagram}

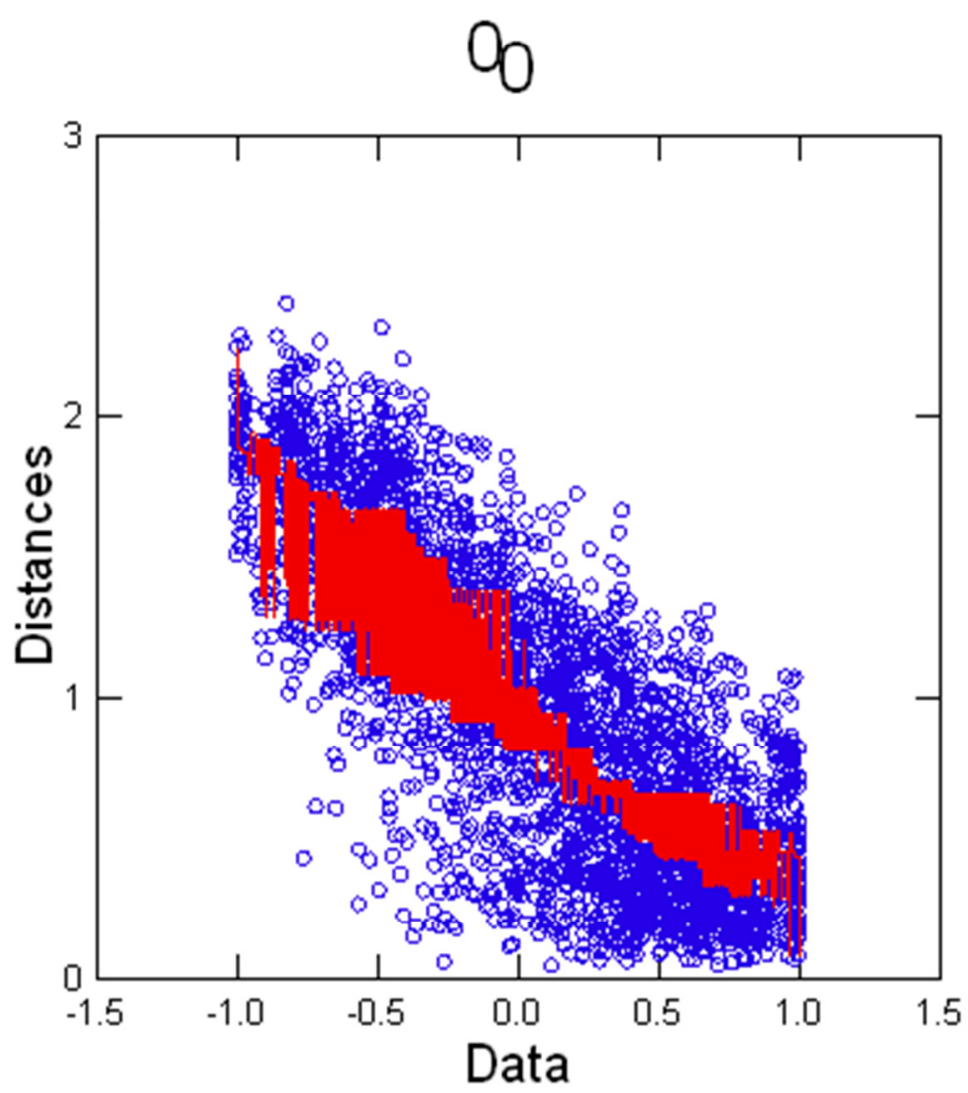

The Shepard Diagram represents the distances between points in the MDS plot against the observed similarities. Ideally these points should adhere to a straight or slightly curved line.

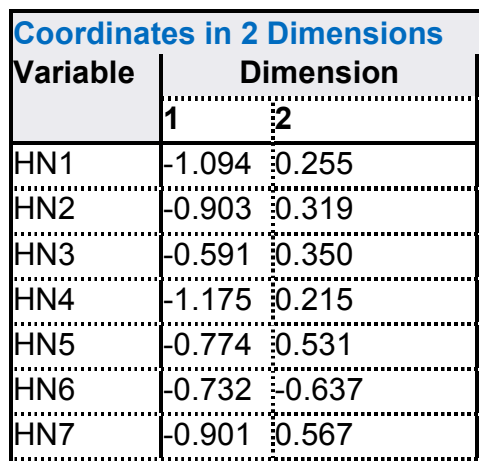

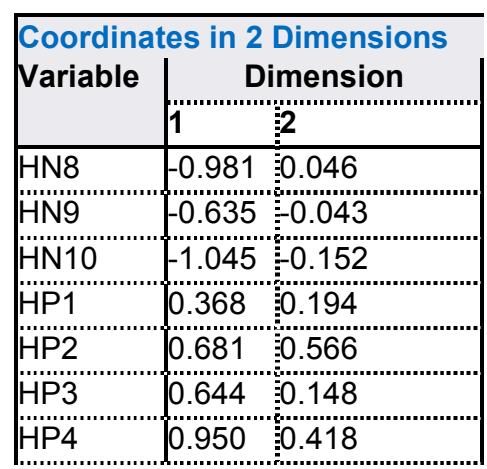

\begin{tabular}{|l|l|l|}
\hline \multicolumn{2}{|l|}{$\begin{array}{l}\text { Coordinates in 2 Dimensions } \\
\text { Variable }\end{array}$} & \multicolumn{1}{c}{ Dimension } \\
& 1 & 2 \\
\hline HP5 & 0.735 & 0.420 \\
\hdashline$H P 6$ & 1.002 & 0.083 \\
\hdashline HP7 & 0.851 & 0.192 \\
\hdashline HP8 & 0.523 & 0.633 \\
\hdashline HP9 & 0.857 & 0.000 \\
\hdashline$H P 10$ & 1.081 & 0.347 \\
\hdashline LN1 & -0.981 & 0.125 \\
\hline
\end{tabular}




\begin{tabular}{|c|c|}
\hline \multicolumn{2}{|c|}{ 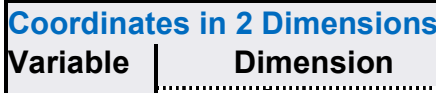 } \\
\hline & 1 \\
\hline LN2 & $-1.076=0.443$ \\
\hline LN33 & $-0.887=0.328$ \\
\hline LN4 & $0.438=0.721$ \\
\hline LN5 & $-0.542=0.553$ \\
\hline L"N6"' & $-0.381=0.357$ \\
\hline Lin7" & $-0.920=0.685$ \\
\hline L"No' & $-1.375-0.182$ \\
\hline LัN9" & $-1.088=0.536$ \\
\hline L"N10 & $-1.099=0.437$ \\
\hline L̈P1 & $1.204=0.151$ \\
\hline
\end{tabular}

\begin{tabular}{|c|c|c|}
\hline $\begin{array}{l}\text { Coordina } \\
\text { Variable }\end{array}$ & tes in & $\begin{array}{l}\text { Dimens } \\
\text { imensio }\end{array}$ \\
\hline & 1 & : \\
\hline LP2 & 0.900 & 0.514 \\
\hline LP3 & 1.081 & 0.076 \\
\hline LP4 & 1.070 & -0.463 \\
\hline LP5 & 0.916 & 0.461 \\
\hline LP6 & 0.800 & $=0.690$ \\
\hline LP7 & 0.947 & -0.397 \\
\hline LP8 & 1.049 & -0.244 \\
\hline LP9 & 1.009 & 0.677 \\
\hline LP10 & 0.949 & 0.245 \\
\hline
\end{tabular}

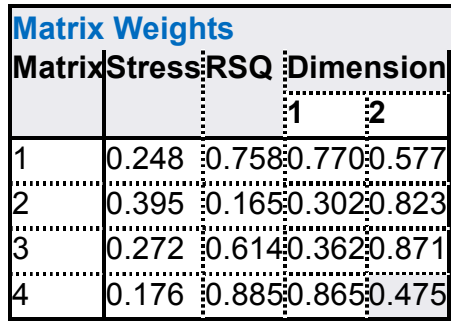

\section{Citation}

Facciani, M. J. (2015). Developing Affective Mental Imagery Stimuli with Multidimensional Scaling. The Quantitative Methods for Psychology, 11 (2), 113-125.

Copyright (C) 2015 Facciani. This is an open-access article distributed under the terms of the Creative Commons Attribution License (CC BY). The use, distribution or reproduction in other forums is permitted, provided the original author(s) or licensor are credited and that the original publication in this journal is cited, in accordance with accepted academic practice. No use, distribution or reproduction is permitted which does not comply with these terms. 